\title{
Integration of mechanotransduction concepts in bone tissue engineering
}

\author{
Dominique P. Pioletti* \\ Laboratory of Biomechanical Orthopedics, Ecole Polytechnique Fédérale de Lausanne, EPFL/STI/IBI/LBO, Station 19, 1015 Lausanne, \\ Switzerland
}

(Received 2 October 2012; final version received 25 February 2013)

\begin{abstract}
Mechanical stimulus has been identified for a long time as a key player in the adaptation of the musculo-skeletal tissues to their function. Mechanical loading is then an intrinsic variable to be considered when new developments are proposed in bone tissue engineering. By combining structural biomechanics and mechanotransduction aspects, a new paradigm is presented for bone tissue engineering. It is proposed that in vivo mechanical loading be used to increased bone formation in the scaffold instead of pre-seeding the scaffold with cells or delivering growth factors. In this article, we demonstrated the feasibility of this approach and compared it to the classical tissue engineering strategy. In particular, we showed that bone formation could be increased in the scaffold that underwent mechanical loading during an in vivo study in rats. A model of bone formation was then proposed to translate the in vivo results into a possible clinical application where the loading of the scaffold would be transmitted by the sharing of the load between an implant and the bone scaffold.
\end{abstract}

Keywords: bone scaffold; total knee implant revision; mechanical loading; in vivo; finite element method; translational medicine

\section{Introduction}

Although bone is known to adapt to its mechanical environment, different kinds of adaptation can be distinguished based on the timescale of their effect. The slowest adaptation process is observed with the size of the jaw during the evolution process of humankind (Figure 1). One million years ago, the size of the Homo erectus jaw was significantly larger than the modern Homo sapiens jaw. We can certainly relate, at least to some extent, this adaptation process to the food quality, which has become softer over the years. The necessity of a powerful jaw then became less important, leading to a continuous decrease in the jaw size over many generations. On a much shorter timescale, throughout our life, the long bones of our skeleton change their mechanical properties from soft cartilage during the foetal period to stiff bone in the adult period, reflecting also the different mechanical constraints these tissues have to face during their development. Finally, on a short timescale, we can observe already after several months that bone adapts its mechanical properties to the presence of an orthopaedic implant, the so-called stress-shielding phenomenon (Huiskes et al. 1992). All these different adaptation phenomena are interesting to study per se; however, if we want to capitalise on the adaptation process for a clinical application, obviously only the short timescale effect of the adaptation can be considered.

The bone adaptation to its mechanical environment has been extensively studied during ex vivo (Stadelmann et al. 2008), or in vivo studies (Rubin and Lanyon 1985) allowing to define effective regimes of mechanically induced bone formation (Goodship et al. 2009). While the mode of action for bone adaptation is still being discussed - fluid flow transport phenomena and/or direct cell stimulation - the mechanosensitive properties of the cells have been widely demonstrated. Direct cell stimulation in the flow chamber has been a powerful approach to highlight the cell sensitivity to the mechanical stimulus (Frangos et al. 1985) and since has been further developed to shed some light on the processes by which cells can sense their mechanical environment (Genetos et al. 2005; Malone et al. 2007). Theoretical developments have also permitted to propose different possibilities of amplification for the sensory role of the cells to the mechanical environment either through coupling effects between fluid and cell deformation (Blecha et al. 2010) or through the deformation of tethering elements that attach the cell process to the canalicular wall (Han et al. 2004).

Surprisingly, despite its central role in the optimal function of bone, adaptation to mechanical stimulation has been only rarely considered as a possible osteoinductor factor for in vivo bone tissue engineering applications (Duty et al. 2007; Boerckel et al. 2009; Roshan-Ghias et al. 2010). Most of the knowledge gathered in mechanotransduction studies has mainly been translated in the development of bioreactor to produce in vitro artificial tissues, e.g. (Sikavitsas et al. 2003; McCoy et al. 2012). Indeed for clinical applications, bone scaffolds are often

\footnotetext{
*Email: dominique.pioletti@epfl.ch 

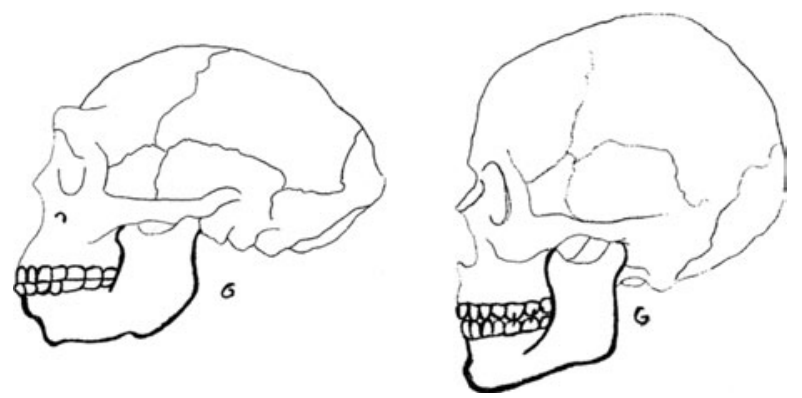

Figure 1. (Left) H. erectus and (right) H. sapiens craniums. A clear adaptation of the jaw size over time can be observed. The powerful jaw of the $H$. erectus has constantly decreased in size to reach the actual one of the modern $H$. sapiens.

facing load-bearing situations. Mechanical loading of the scaffold is then an intrinsic part of the clinical situation.

As described in a recent review, two aspects can be distinguished in the mechanical loading of the scaffold (Pioletti 2010). First, the scaffold has to sustain its mechanical environment. Structural biomechanical analysis has then to be considered by computing the stress field in the scaffold. Second, the mechanical loading can be used to favour bone formation in the scaffold. Mechanotransduction aspects then need to be introduced. If correctly combined, these two aspects (structural biomechanics and mechanotransduction) could then transform the properties of a biomaterial from osteoconductive to osteoinductive ones.

Although the classical concept in tissue engineering relies on the three pillars (scaffold, cell and growth factor), an alternative paradigm could be proposed by considering the mechanical stimulus as a key part in tissue engineering. In this article, we demonstrated the feasibility of this approach and compared it to the classical tissue engineering strategy.

\section{Classical bone tissue engineering}

\subsection{Clinical applications}

The function of the skeleton being mostly to mechanically support the body, it is then not surprising that scaffolds used for bone tissue engineering face load-bearing situations. For the sake of illustration, we focus our attention on the tibia, considering a tibial osteotomy and a revision of total knee implant for our targeted clinical applications (Figure 2). In these two situations, the possible combination of structural biomechanics and mechanotransduction aspects can be exemplified for the development of an artificial bone scaffold. In particular, the classical schema for bone tissue engineering should consider structural biomechanics for the scaffold development (Figure 3(a)).
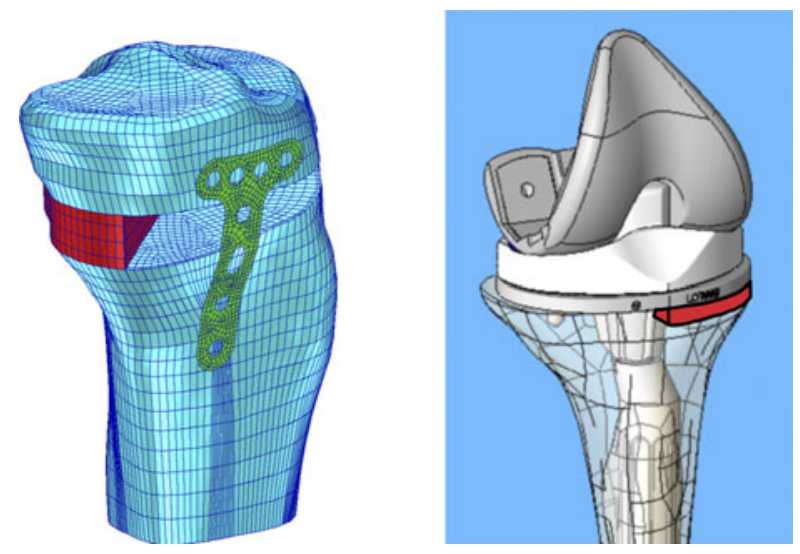

Figure 2. (Left) Finite element model of a tibial osteotomy and (right) schematic representation of a revision of total knee arthroplasty. In both situations, the artificial bone scaffold is represented in red. A load sharing between the scaffold and the implant (plate for osteotomy and tibial insert for total knee arthroplasty) could be obtained and used as mechanical stimulus for improving bone formation in the scaffold.

\subsection{Scaffold}

The definition of the clinical applications allows us to define the corresponding mechanical and geometrical specification for the artificial bone scaffold. A structural biomechanical analysis could then be followed in order to define the mechanical parameters, such as elastic modulus,

(a)
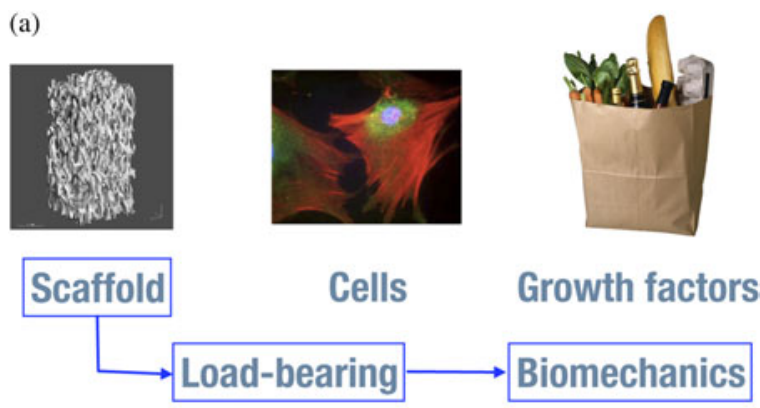

(b)

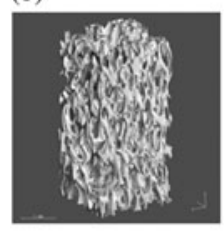

Scaffold

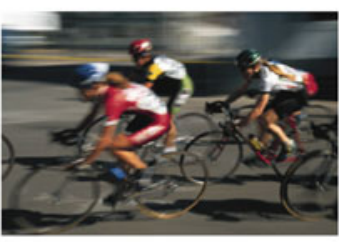

Biomechanics

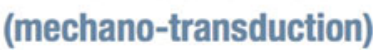

Figure 3. (a) Classical schema for bone tissue engineering with the specific considerations of structural biomechanics for the scaffold development. (b) In vivo biomechanical stimulation of the scaffold is proposed to increase bone formation through mechanotransduction aspects and could reduce the need of preseeding cells or growth factors in the scaffold. 


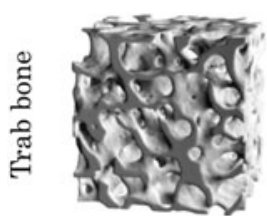

Plate-like

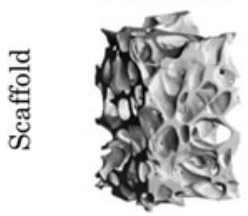

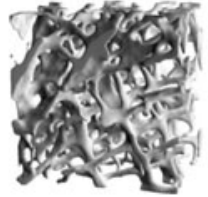

Hybrid

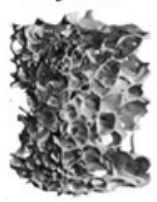

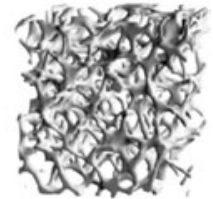

Rod-like

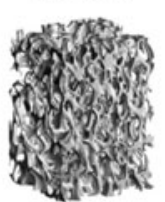

Figure 4. Different morphological scaffolds of PLA reinforced with $\beta$-TCP particles were obtained using a supercritical $\mathrm{CO}_{2}$ foaming technique. Natural trabecular bone morphologies are shown for comparison. Source: Adapted from Mathieu et al. (2006).

or strength and geometrical parameters, such as shape or size of the artificial scaffold. For the scaffold to be used for revision of total knee arthroplasty, based on the material properties of polylactic acid (PLA) scaffold presenting $80 \%$ porosity, the finite element method (FEM) analysis showed that failure (defined as calculated strain exceeding the experimental ultimate strain of the scaffold) was predicted only for scaffolds thinner than $5 \mathrm{~mm}$ (Terrier et al. 2009). In parallel, this kind of study also allows us to estimate that an elastic modulus of 50-100 MPa would be enough for the tibial osteotomy application (Blecha et al. 2005). Based on this information, a PLA-based scaffold reinforced by $\beta$-TCP particles of $5 \mathrm{wt} \%$ has been developed through a (solvent-free) super-critical $\mathrm{CO}_{2}$ foaming technique (Mathieu et al. 2006). In addition to the targeted mechanical properties, depending on the physical parameters used during the foaming process, the obtained scaffolds mimic the different natural morphologies of trabecular bone (Figure 4).

After being extensively tested through in vitro (Montjovent et al. 2005) and in vivo tests in rats (Montjovent et al. 2007), the developed scaffold was inserted in sheep distal femur and proximal tibia (van der Pol et al. 2010). At 12 months, it was observed that new bone had invaded the scaffold, even reaching its centre (Figure 5). However, the bone ingrowth rate might be considered slow as after 4 months, only moderate bone formation was observed in the scaffold (bone volume BV/ total volume TV reaching 4\%). From the in vivo evaluation in rat and sheep, it could be concluded that the developed scaffold presented osteoconductor properties.

\subsection{Foetal cell therapy}

As suggested in Figure 3(a), the addition of cells might increase the bone formation rate in the scaffold. In our particular situation, we use human bone foetal cells

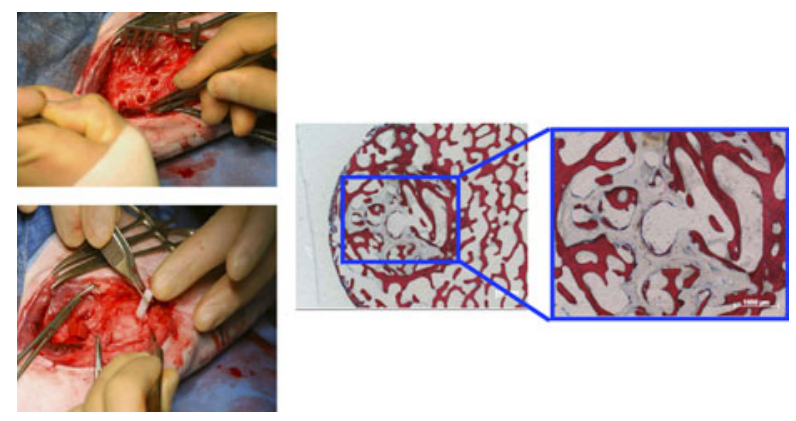

Figure 5. Sheep model used to evaluate the ostoinduction properties of the developed scaffold. After 12 months, bone ingrowth was also observed in the centre of the scaffold. Source: Adapted from van der Pol et al. (2010).

obtained from a single organ donation as cell sources (Pioletti et al. 2006). We implanted the developed scaffold with or without foetal cells in rat condyles and observed a $100 \%$ increase in bone formation after 2 months when foetal cells are associated with the scaffold (Montjovent et al. 2008). The presence of foetal cells allows the transformation of the developed scaffold from an osteoconductor material into an osteoinductor material. Obviously, this is a very positive result also obtained with other cell types as well, e.g. (Anselme et al. 1999; Stevens et al. 2005). The use of cells for tissue engineering comes with a drawback. Indeed, the combination of a scaffold with cells drastically complicates the regulatory aspects. It is then a long and costly process to get an approval for this kind of product. An alternative strategy to transform the developed scaffold into an osteoinductive material could be proposed.

\section{New paradigm in bone tissue engineering}

Combining structural biomechanics and mechanotransduction aspects could be an elegant method of capitalising on the intrinsic load-bearing situation arising in bone tissue engineering applications (Figure 3(b)). This combination has been formally described in the development of an artificial bone scaffold by optimising properties such as elastic modulus, permeability, Poisson coefficient and porosity to maximise the fluid exchange in the scaffold while keeping the flow induced-shear stress at an osteoinductor level (Blecha et al. 2009). The knowledge of the value of the mechanical stimulus is then central to obtain an increase in bone formation in the scaffold. This value shows the bone as being dependent in a non-linear way on the deformation values and number of daily loading cycles (Ozcivici et al. 2010). For example, a mechanical stimulus of $1000 \mu \varepsilon$ applied a 100 times per day stimulated bone formation in turkey ulna.

The use of mechanical loading to increase bone formation in the scaffold has been proposed (Duty et al. 


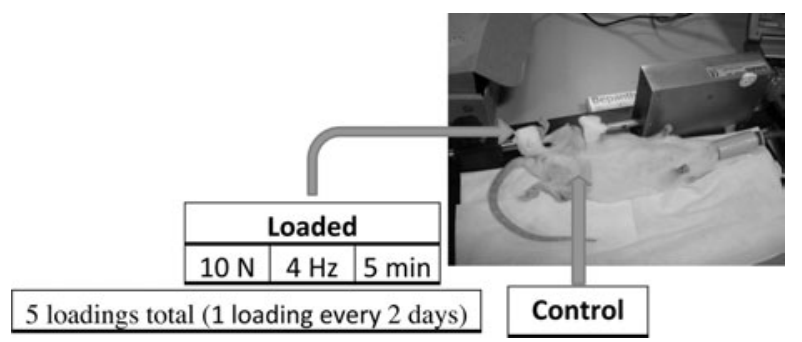

Figure 6. An in vivo mechanical stimulus device to evaluate the effect of mechanical loading on bone formation in the scaffolds implanted in rat condyles. Source: Adapted from Roshan-Ghias et al. (2010).

2007). In the study of Duty et al., the scaffolds were seeded with mesenchymal stromal cells prior to implantation, and the loading was percutaneously applied rendering a very difficult translation to a clinical application. We have recently proposed to apply a mechanical stimulus to an implanted scaffold in a less invasive way and without any pre-seeding of cells. The scaffolds were inserted in rat condyle, and the knee was stimulated using a compression machine previously developed (Stadelmann et al. 2008) (Figure 6). The right leg of each animal was loaded and left leg was kept unloaded as control. To determine a loading regime that would induce bone formation in the relationship presented in Ozcivici et al. (2010), a finite element model of the in vivo loading was developed (Roshan-Ghias et al. 2010). The theoretically determined mechanically induced bone formation corresponded to a $10 \mathrm{~N}$ loading at $4 \mathrm{~Hz}$ for $5 \mathrm{~min}$. To not excessively disturb the rat, this loading regime was applied every 2 days during 10 days starting 14 days after the implantation of the scaffold. Interestingly, the $10 \mathrm{~N}$ loading resulted in strains of approximately $620 \mu \varepsilon$, which is close to the strains induced in the human tibia while walking (Burr et al. 1996).

To follow the effect of the mechanical stimulus on the inserted scaffolds, a longitudinal $\mu \mathrm{CT}$ study was performed to evaluate the bone formation (bone volume over total volume, BV/TV) between the specifically loaded and non-loaded scaffolds. The animals were scanned seven times at regular intervals until 250 days post-implantation. The positive effect of the mechanical stimulation on the bone formation in the scaffold was clearly established (Figure 7). Indeed, by using a recently developed dynamical histomorphometry analysis (Schulte et al. 2011), the mechanical stimulus was shown to favour bone formation, while decreasing bone resorption over time in the scaffold (Roshan-Ghias et al. 2011).

The obtained in vivo results allow us to conclude that the use of an appropriate mechanical stimulus can transform the scaffold from an osteoconductor material into an osteoinductor material. In the context of tissue engineering, we could then propose a new paradigm by

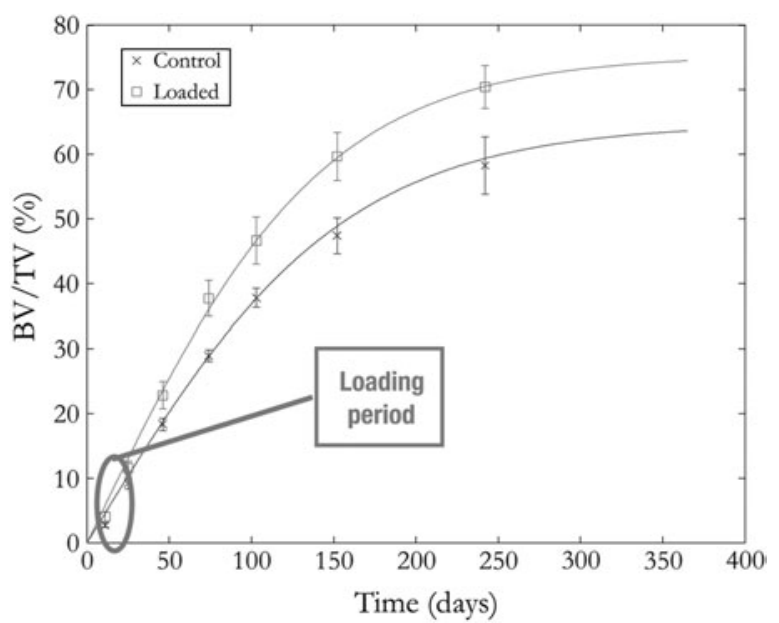

Figure 7. Comparison of BV/TV in the loaded and non-loaded scaffold at different time points. As a reminder, the duration of the loading is indicated. The BV/TV was significantly increased by $18 \%$ in the loaded scaffold versus the non-loaded one $(n=6$ and $p<0.05)$. Source: Adapted from Roshan-Ghias, Lambers et al. (2011).

replacing the use of cells and growth factors with mechanical stimulus when the latter can be controlled in specific clinical applications. This new paradigm presents the advantage of easing the aspects of regulatory affairs and may also induce an optimised bone formation with respect to the mechanical performances the scaffold has to fulfil.

\section{Translation of biomechanical stimulation into a clinical tissue engineering application}

To benefit from the new proposed paradigm, we have to define clinical applications where the mechanical stimulus can be controlled. Revision of total knee arthroplasty could be a possible application.

To translate results obtained with a rat condyle study into useable information for a revision of total knee arthroplasty situation, a model of bone formation using a scaffold would be of great help. There are two different approaches to model the bone formation in a scaffold. The first one is to describe the different processes involved in the bone formation from cell migration, cell differentiation, extracellular matrix production and mineralisation to name only a few. This approach allowed, for example, to evaluate the effect of pre-seeding cells in the scaffold on tissue formation (Checa and Prendergast 2010). New hypothesis or knowledge in tissue formation can be generated with these kinds of models. However, these models are difficult to use for prediction of bone formation in particular situations as they necessitate many parameters usually difficult to identify with an experimental data. A second approach to describe bone formation in 
scaffold is through a phenomenological model. In this approach, no information is provided on the processes of how bone is formed in a scaffold, only the predictive capacity of the model to anticipate bone formation is targeted. Phenomenological models used a minimal number of parameters. A phenomenological description has been proposed by observing that bone formation followed a diffusion phenomenon in scaffolds (RoshanGhias et al. 2011). A corresponding diffusion model was developed and allowed to catch the in vivo spatial and temporal evolution of bone formation in scaffold. The degradation of the scaffold was supposed to be slow enough so that a transfer of the mechanical load could be obtained from the scaffold to the newly formed bone. The prediction capability of the model was validated with different published experimental data. Two sets of parameters appearing in the diffusion model could be identified with the in vivo bone formation data of the rat condyle. The first set was obtained for the non-loaded situation of the scaffold and the second set corresponded to the loaded situation. By assuming that these two sets of parameters obtained with rat data could be used to predict bone formation in scaffold implanted in human bone, a prediction of bone formation in scaffold used in revision of total knee arthroplasty was calculated for two situations: a loaded scaffold and a non-loaded scaffold, using the corresponding two sets of identified parameters of the diffusion model. The loaded scaffold corresponded to a situation where a load sharing is obtained between the tibial implant and the scaffold placed below. A finite element model of a total knee arthroplasty revision (Figure 2) was developed and combined with the diffusion model of bone formation in scaffold (Roshan Ghias et al. in press). At 24 months, a $300 \%$ increase in the mechanical properties induced by a corresponding increase in bone formation in the scaffold was predicted when mechanical loading was applied. These results demonstrated the potential of using biomechanical stimulus for bone tissue engineering applications.

Obviously, more experimental data would be necessary to confirm the feasibility of using mechanical loading as an osteoinductor factor in scaffolds for clinical applications. In particular, it would be important to precisely determine the amount of load needed to be transmitted by the implant to the scaffold. In parallel, we could also imagine to have loads applied on the scaffold during controlled exercises performed during physiotherapy sessions post-surgery.

\section{Conclusions}

The actual paradigm in tissue engineering is to combine a scaffold with cells and/or growth factors. This strategy, while demonstrating its pertinence in multiple in vivo studies, hardly leads to clinical applications as recently reviewed specifically for scaffold used in bone tissue engineering applications (Hollister and Murphy 2011). In parallel, the importance of mechanical stimulation on the behaviour of biomaterials has been identified since several years. For example, the absence of mechanical loading has been shown to affect the osteoconduction of the wellaccepted biomaterials such as calcium phosphate granules (Handschel et al. 2002). The combination of scaffold and in vivo controlled mechanical stimulation could then represent a complementary strategy to the classical tissue engineering paradigm.

\section{Acknowledgements}

This project was supported by SNSF Grants (Nos 205320121893, 32003B-108386 and 404640-101114) and the InterInstitutional Center for Translational Biomechanics EPFLCHUV-DAL. The author would like to thank Guillaume Pioletti for his drawings of craniums in Figure 1.

\section{References}

Anselme K, Noel B, Flautre B, Blary MC, Delecourt C, Descamps M, Hardouin P. 1999. Association of porous hydroxyapatite and bone marrow cells for bone regeneration. Bone. 25(2):51S-54S.

Blecha LD, Rakotomanana L, Razafimahery F, Terrier A, Pioletti DP. 2009. Targeted mechanical properties for optimal fluid motion inside artificial bone substitutes. J Orthop Res. 27(8):1082-1087.

Blecha LD, Rakotomanana L, Razafimahery F, Terrier A, Pioletti DP. 2010. Mechanical interaction between cells and fluid for bone tissue engineering scaffold: modulation of the interfacial shear stress. J Biomech. 43(5):933-937.

Blecha LD, Zambelli PY, Ramaniraka NA, Bourban PE, Månson JA, Pioletti DP. 2005. How plate positioning impacts the biomechanics of the open wedge tibial osteotomy: a finite element analysis. Comput Methods Biomech Biomed Eng. 8(5):307-313.

Boerckel JD, Dupont KM, Kolambkar YM, Lin AS, Guldberg RE. 2009. In vivo model for evaluating the effects of mechanical stimulation on tissue-engineered bone repair. $\mathrm{J}$ Biomech Eng. 131(8):084502.

Burr DB, Milgrom C, Fyhrie D, Forwood M, Nyska M, Finestone A, Hoshaw S, Saiag E, Simkin A. 1996. In vivo measurement of human tibial strains during vigorous activity. Bone. 18(5): 405-410.

Checa S, Prendergast PJ. 2010. Effect of cell seeding and mechanical loading on vascularization and tissue formation inside a scaffold: a mechano-biological model using a lattice approach to simulate cell activity. J Biomech. 43(5):961-968.

Duty AO, Oest ME, Guldberg RE. 2007. Cyclic mechanical compression increases mineralization of cell-seeded polymer scaffolds in vivo. J Biomech Eng. 129(4):531-539.

Frangos JA, Eskin SG, Mclntire LV, Ives CL. 1985. Flow effects on prostacyclin production by cultured human endothelial cells. Science. 227(4693):1477-1479. 
Genetos DC, Geist DJ, Liu D, Donahue HJ, Duncan RL. 2005. Fluid shear-induced ATP secretion mediates prostaglandin release in MC3T3-E1 osteoblasts. J Bone Miner Res. 20(1):41-49.

Goodship AE, Lawes TJ, Rubin CT. 2009. Low-magnitude highfrequency mechanical signals accelerate and augment endochondral bone repair: preliminary evidence of efficacy. J Orthop Res. 27(7):922-930.

Han Y, Cowin SC, Schaffler MB, Weinbaum S. 2004. Mechanotransduction and strain amplification in osteocyte cell processes. Proc Natl Acad Sci USA. 101(47): 16689-16694.

Handschel J, Wiesmann HP, Stratmann U, Kleinheinz J, Meyer U, Joos U. 2002. TCP is hardly resorbed and not osteoconductive in a non-loading calvarial model. Biomaterials. 23(7):1689-1695.

Hollister SJ, Murphy WL. 2011. Scaffold translation: barriers between concept and clinic. Tissue Eng Part B Rev. 17(6):459-474.

Huiskes R, Weinans H, van Rietbergen B. 1992. The relationship between stress shielding and bone resorption around total hip stems and the effects of flexible materials. Clin Orthop. 274:124-134.

Malone AM, Anderson CT, Tummala P, Kwon RY, Johnston TR, Stearns T, Jacobs CR. 2007. Primary cilia mediate mechanosensing in bone cells by a calcium-independent mechanism. Proc Natl Acad Sci USA. 104(33):13325-13330.

Mathieu LM, Mueller TL, Bourban PE, Pioletti DP, Müller R, Månson JAE. 2006. Architecture and properties of anisotropic polymer composite scaffolds for bone tissue engineering. Biomaterials. 27(6):905-916.

McCoy RJ, Jungreuthmayer C, O'Brien FJ. 2012. Influence of flow rate and scaffold pore size on cell behavior during mechanical stimulation in a flow perfusion bioreactor. Biotechnol Bioeng. 109(6):1583-1594.

Montjovent MO, Mark S, Mathieu L, Scaletta C, Scherberich A, Delabarde C, Zambelli PY, Bourban PE, Applegate LA, Pioletti DP. 2008. Human fetal bone cells associated with ceramic reinforced PLA scaffolds for tissue engineering. Bone. 42(3):554-564.

Montjovent MO, Mathieu L, Hinz B, Applegate LL, Bourban PE, Zambelli PY, Manson JA, Pioletti DP. 2005. Biocompatibility of bioresorbable poly(l-lactic acid) composite scaffolds obtained by supercritical gas foaming with human fetal bone cells. Tissue Eng. 11(11-12):1640-1649.

Montjovent MO, Mathieu L, Schmoekel H, Mark S, Bourban PE, Zambelli PY, Laurent-Applegate LA, Pioletti DP. 2007. Repair of critical size defects in the rat cranium using ceramic-reinforced PLA scaffolds obtained by supercritical gas foaming. J Biomed Mater Res A. 83(1):41-51.
Ozcivici E, Luu YK, Adler B, Qin YX, Rubin J, Judex S, Rubin CT. 2010. Mechanical signals as anabolic agents in bone. Nat Rev Rheumatol. 61:50-59.

Pioletti DP. 2010. Biomechanics in bone tissue engineering. Comput Methods Biomech Biomed Eng. 13(6):837-846.

Pioletti DP, Montjovent MO, Zambelli PY, Applegate L. 2006. Bone tissue engineering using foetal cell therapy. Swiss Med Wkly. 136(35-36):557-560.

Roshan-Ghias A, Lambers FM, Gholam-Rezaee M, Müller R, Pioletti DP. 2011. In vivo loading increases mechanical properties of scaffold by affecting bone formation and bone resorption rates. Bone. 49(6):1357-1364.

Roshan-Ghias A, Terrier A, Bourban PE, Pioletti DP. 2010. In vivo cyclic loading as a potent stimulatory signal for bone formation inside tissue engineering scaffold. Eur Cell Mater. 19:41-49.

Roshan-Ghias A, Terrier A, Jolles BM, Pioletti DP. in press. Translation of biomechanical concepts in bone tissue engineering: from animal study to revision knee arthroplasty. Comput Methods Biomech Biomed Eng. doi:10.1080/ 10255842.2012.719607.

Roshan-Ghias A, Vogel A, Rakotomanana L, Pioletti DP. 2011. Prediction of spatio-temporal bone formation in scaffold by diffusion equation. Biomaterials. 32(29):7006-7012.

Rubin CT, Lanyon LE. 1985. Regulation of bone mass by mechanical strain magnitude. Calcif Tissue Int. 37(4):411-417.

Schulte FA, Lambers FM, Kuhn G, Muller R. 2011. In vivo micro-computed tomography allows direct three-dimensional quantification of both bone formation and bone resorption parameters using time-lapsed imaging. Bone. 48(3):433-442.

Sikavitsas VI, Bancroft GN, Holtorf HL, Jansen JA, Mikos AG. 2003. Mineralized matrix deposition by marrow stromal osteoblasts in 3D perfusion culture increases with increasing fluid shear forces. Proc Natl Acad Sci USA. 100(25):14683-14688.

Stadelmann VA, Terrier A, Pioletti DP. 2008. Microstimulation at the bone-implant interface upregulates osteoclast activation pathways. Bone. 42(2):358-364.

Stevens MM, Marini RP, Schaefer D, Aronson J, Langer R, Shastri VP. 2005. In vivo engineering of organs: the bone bioreactor. Proc Natl Acad Sci USA. 102(32):11450-11455.

Terrier A, Sedighi-Gilani M, Roshan-Ghias A, Aschwanden L, Pioletti DP. 2009. Biomechanical evaluation of porous biodegradable scaffolds for revision knee arthroplasty. Comput Methods Biomech Biomed Eng. 12(3):333-339.

van der Pol U, Mathieu L, Zeiter S, Bourban PE, Zambelli PY, Pearce SG, Bouré LP, Pioletti DP. 2010. Augmentation of bone defect healing using a new biocomposite scaffold: an in vivo study in sheep. Acta Biomater. 6(9):3755-3762. 